\title{
A new technique for evaluating mesospheric momentum balance utilizing radars and satellite data
}

\author{
D. J. Frame ${ }^{1}$, B. N. Lawrence ${ }^{1}$, G. J. Fraser ${ }^{1}$, R. A. Vincent ${ }^{2}$, A. Dudhia ${ }^{2}$ \\ ${ }^{1}$ Department of Physics and Astronomy, University of Canterbury, New Zealand \\ 2 Department of Physics, University of Adelaide, Australia \\ ${ }^{3}$ Department of Physics, University of Oxford, United Kingdom
}

Received: 12 May 1999 / Revised: 15 November 1999 / Accepted: 30 November 1999

\begin{abstract}
A new method for evaluating momentum balance in the mesosphere using radar and satellite data is presented. This method is applied to radar wind data from two medium frequency installations (near Adelaide, Australia and Christchurch, New Zealand) and satellite temperature data from the Improved Stratospheric and Mesospheric Sounder (ISAMS). Because of limitations in data availability and vertical extent, the technique can only be applied to evaluate the momentum balance at $80 \mathrm{~km}$ above the radar sites for May 1992. The technique allows the calculation of the residual terms in the momentum balance which are usually attributed to the effects of breaking gravity waves. Although the results are inconclusive above Adelaide, this method produces values of zonal and meridional residual accelerations above Christchurch which are consistent with expectation. In both locations it is apparent that geostrophic balance is a poor approximation of reality. (This result is not dependent on a mismatch between the radar and satellite derived winds, but rather is inherent in the satellite data alone.) Despite significant caveats about data quality the technique appears robust and could be of use with data from future instruments.
\end{abstract}

Key words: Meteorology and atmospheric dynamics (middle atmosphere dynamics; waves and tides; instruments and techniques)

\section{Introduction}

It is well known that the influence of gravity wave breaking is important in the momentum balance of the mesosphere (Lindzen, 1981). However, direct measure-

Correspondence to: B. N. Lawrence

e-mail: b.lawrence@phys.canterbury.ac.nz ments of the effects of gravity wave breaking are often difficult to obtain: while balloons, lidars and some radars can resolve the structure of individual gravity waves over a site; their effects on the background flow as they break usually requires numerical parameterization. The broad viewing region possessed by satellite instruments is generally unhelpful in this regard, too, the effects of averaging across the viewing region effectively smear out evidence of gravity wave effects. In this work we introduce a new method which makes use of both radar and satellite data to calculate the effect of gravity wave breaking on momentum balance in the mesosphere.

The momentum equations appropriate for large-scale fluid flow on a sphere can be written in pressure coordinates as

$$
\begin{aligned}
\frac{\partial u}{\partial t}+ & {\left[\frac{u}{a \cos \phi} \frac{\partial u}{\partial \lambda}+\frac{v}{a \cos \phi} \frac{\partial(u \cos \phi)}{\partial \phi}\right] } \\
& +w \frac{\partial u}{\partial z}-f v+\frac{1}{a \cos \phi} \frac{\partial \Phi}{\partial \lambda}=D
\end{aligned}
$$

and

$$
\begin{aligned}
\frac{\partial v}{\partial t} & +u^{2} \frac{\tan \phi}{a}+\left[\frac{u}{a \cos \phi} \frac{\partial v}{\partial \lambda}+\frac{v}{a} \frac{\partial v}{\partial \phi}\right] \\
& +w \frac{\partial v}{\partial z}+f u+\frac{1}{a} \frac{\partial \Phi}{\partial \phi}=E
\end{aligned}
$$

where $u, v$ and $w$ are the velocity components relative to longitude, $\lambda$, latitude, $\phi$, and $\log$ pressure height $z=-H \ln P / P_{0}$. The Coriolis parameter, $f=2 \Omega \sin \phi$ where $\Omega$ is the Earth's rotation rate, $a$ is the Earth radius and $\Phi$ the geopotential height of the pressure surface.

In these equations, the first term represents the local acceleration, the terms in the square brackets are the horizontal advection terms, which are followed by the vertical advection term, the Coriolis acceleration and the pressure gradient terms. The $u^{2}$ term is part of the meridional advection term which arises from the convergence of the meridians (and is usually only important near the poles and where there are very 
strong winds). The right hand side of each equation ( $D$ and $E$ respectively) represents the force per unit mass due to unresolved phenomena, which in the mesosphere we believe to be primarily the effect of breaking gravity waves (but other phenomena such as tidal contamination along with measurement error will also contribute to these terms).

We use wind data from two medium frequency (MF) radar facilities and V10 temperature data from the Improved Stratospheric and Mesospheric Sounder (ISAMS) (Dudhia and Livesey, 1996) aboard the Upper Atmosphere Research Satellite (UARS) to show how we can calculate the $D$ and $E$ terms in these equations above the radar locations. This calculation is carried out at an altitude of $80 \mathrm{~km}$, which is the only height where sufficient radar data is available and for which ISAMS temperatures are simultaneously available. The two radar sites are Birdlings Flat $\left(44^{\circ} \mathrm{S}, 173^{\circ} \mathrm{E}\right)$, near Christchurch, New Zealand and Adelaide, Australia $\left(35^{\circ} \mathrm{S}, 138^{\circ} \mathrm{E}\right)$. The analysis is carried out for May 1992 which is the only time during successful ISAMS operation when data from both radar sites was available.

We begin by outlining the techniques used, then present our results, and conclude with a discussion of the material.

\section{Numerical techniques and data}

To evaluate the momentum balance from the available data it is necessary to evaluate the terms in Eq. (1) and (2). With appropriate approximations and assumptions, satellite data can be used to evaluate all these terms. However, previous workers have been limited to evaluating these equations in the zonal mean (e.g. Hamilton, 1983; Smith and Lyjak, 1985; Marks, 1989, and others). The technique of Marks (1989) can, in principle, be used to evaluate the terms in all three dimensions but in practice it is very difficult to make the numerics remain stable.

The Marks (1989) method is based on iterating around a loop involving the thermodynamic equation, the mass continuity equation and the two momentum equations. Using this method an estimate of the vertical wind $(w)$ is obtained, but because of the lack of a fifth dynamical equation, this method requires that $E$ be set to zero in order to solve for the zonal wind. Here, by using independent measures of the geopotential gradient and the horizontal wind, we do not need this requirement. However, we do need to assume that the terms involving vertical advection and time derivatives are negligible. This latter assumption can be justified to some extent by using monthly means, and is amenable to assessment.

Our method of solution is to evaluate the terms which are "known" (those on the left hand side) and then evaluate $D$ and $E$ as the residuals remaining when the terms on the left hand side are summed. Here we use the radar data wherever possible, but the curvature terms (those in the square brackets of Eqs. 1 and 2) cannot be calculated from single station data. Here we assume that while the radar wind is not the same as the winds derived from the satellite measurements, the wind gradients are the same. Thus, neglecting the time derivatives and the vertical motion terms, and using the geopotential gradients from the satellite data, it is possible to evaluate all the terms on the left hand side and hence calculate the residuals.

We have used geopotential heights from the UK Meteorological Office's Assimilated data product (Swinbank and O'Neill, 1994) at $100 \mathrm{hPa}$ and then stacked gridded ISAMS temperature thicknesses on top of these. The level 3ATISAMS data was gridded using the procedure described in Rosier et al. (1994). To calculate the gradients in the wind fields, we have calculated the gradient of the geostrophic wind fields obtained from these geopotential height fields.

Both the satellite data and the radar data can be severely contaminated by tidal influences. By using monthly means, we believe we have removed tidal influences in the satellite data because the UARS satellite has precessed through nearly a full day in that time. As far as the radar data is concerned, we have binned the available data into hourly bins, and then averaged those for the daily and then monthly values. Because each hourly bin is given equal weight, the diurnal variations should not lead to tidal biases in the resulting mean wind (see also Frame et al., submitted 1999).

There are other major potential problems which need to be considered in an analysis of this kind. Firstly, the satellite data is from the highest retrieval level, and at a time when the instrument was not functioning with the highest possible signal-to-noise ratio. These effects mean that the geopotential heights are probably more inaccurate than they might normally be. Given that we need to differentiate these fields using finite differences, there is a potential problem there. In order to examine the effect of this problem, we repeat our analysis using geopotential height fields which have been smoothed (using a nearest point, 1/4, 1/2,1/4 filter in both $x$ and $y$ ) before calculating their differences. In this repeated analysis we have also used the same filter to smooth the geostrophic wind fields before calculating the differences of those fields.

A second potential problem is that the MF radar data are known to exhibit some differences from other wind measuring techniques (e.g. Burrage et al., 1996; Khattatov et al., 1996). It is not obvious that these differences reflect a poor wind measurement per se, but if the radar is not measuring the wind accurately, it will obviously lead to errors in the calculations we present. In an effort to assess the potential effect of such errors, we have examined data from a comprehensive comparison of the Birdlings Flat winds with HRDI observations (Frame et al., 1999). That comparison showed a tendency for the Birdlings Flat radar winds to be more easterly than the HRDI winds. We have then produced a regression for the $80 \mathrm{~km}$ winter winds between the Birdlings Flat radar data and the HRDI data discussed in Frame et al. (1999) giving 
$u_{c}=0.72 u_{r}+16$

where $u_{c}$ is the corrected zonal wind and $u_{r}$ is the actual measured radar wind. We have then used this regression line to estimate a possible correction to the Birdlings Flat zonal wind to bring them more in line with the HRDI measurements. (The meridional wind was found to be generally in good agreement with HRDI and is not corrected.) These corrected zonal winds can be used to produce a set of results which should be interpreted as an indicator of the scale of potential errors associated with any bias in the MF radar winds (if such a bias is real, see Frame et al., 1999, for a discussion of the issues for these data).

It would be possible to carry out such a correction procedure for Adelaide, but as will be seen, it is not obvious that such a procedure is worthwhile there. We simply include it for Birdlings Flat to show the potential scale of any problems arising from biases in the radar data.

\section{Results}

\subsection{Mean winds}

Geostrophic balance allows one to calculate winds from geopotential height gradients, but whether such winds are a real reflection of the actual wind depends critically on the assumptions used. In particular, we know that if there is much local curvature in the flow, geostrophic winds will be an overestimate of the actual wind (Randel, 1987). We also know that if there is significant gravity wave driving ( $D$ and $E$ of Eqs. 1 and 2 significantly non-zero), then geostrophic winds will also not be close to the true wind (and might be either smaller or larger, depending on the sign of $D$ and/or $E$ ). Thus, mismatches between actual winds and calculated geostrophic winds are evidence of curvature and/or gravity wave driving.

The daily mean winds from the Birdlings Flat radar are used to produced monthly mean values from $80 \mathrm{~km}$ for May 1992 which are tabulated in Table 1. Also tabulated are the monthly mean winds produced from the daily values of the geostrophic winds produced as described already. It can be seen that there are significant discrepancies in the mean winds, although the standard deviations do include a wide range. The discrepancy between the zonal winds can be mitigated if the radar zonal wind is corrected using equation 3 , but the value $u_{c}=25$ is still significantly less than the geostrophic wind, which indicates that curvature and

Table 1. Monthly mean Birdlings Flat radar and geostrophic wind vectors and associated standard deviations for May 1992

\begin{tabular}{lrr}
\hline Wind type & $\langle\mathbf{u}\rangle$ & $\sigma$ \\
\hline$u_{r}$ & 10 & 10 \\
$u_{g}$ & 34 & 43 \\
$v_{r}$ & 3 & 8 \\
$v_{g}$ & 10 & 58 \\
\hline
\end{tabular}

or gravity wave driving could be contributing to the momentum balance. Other possible reasons for the discrepancy include all the factors outlined in Frame et al. (1999) and the fact that the ISAMS data is at the very top of its operational range where one would expect the temperature retrievals to significantly underestimate the true deviations from the model climatology.

In addition, one needs to remember that the satellite instrument and the radar are sampling different volumes of the atmosphere. The viewing volume for the ISAMS instrument is vastly different from that of the radar, and the procedure used to grid the temperature data and produce the geopotential fields involves a large amount of smoothing and interpolation. After all that, the ISAMS winds need to be derived from the geopotential field, and then interpolated to the radar location. One might expect then that the geostrophic wind fields might be reliable on very large scales (given that the satellite data are the smoothed gridded results of large sampling volumes) but are not necessarily representative of the smaller scales such as those sampled by the radar.

However, notwithstanding all these caveats, much of the difference in these wind measurements could be due to curvature and gravity wave driving, and amenable to the analysis which follows.

\subsection{Equation residuals}

We cannot evaluate all the terms on the left hand side of Eqs. (1) and (2) directly. As explained earlier, we assume that the terms involving the time derivatives and vertical motion are negligible, and we use the satellite data to evaluate the horizontal gradients of the winds needed to evaluate the horizontal advection terms. In the tables that follow we denote these horizontal advective terms as

$u \frac{\partial u}{\partial x}, v \frac{\partial c u}{\partial y}, u \frac{\partial v}{\partial x}$ and $v \frac{\partial v}{\partial y}$

where $c$ is an abbreviation for $\cos \theta$, but they are calculated using fourth order derivatives on the full spherical grid. Note that in the tables $\tan \theta$ is also abbreviated to $t$.

For each equation we proceed by evaluating the terms at each vertex of the grid around the radar site, assuming that the radar wind is constant across the grid, but using the appropriate gradient terms from the full grid at those positions. We can use the momentum balance for the vertices to allow us to obtain an estimate of the behaviour of our solution in the neighbourhood of the radar. We then calculate the solution above the radar by interpolating the satellite values to the radar location.

As a check on the solutions, we can then proceed to modify the values of $u_{r}$ and $v_{r}$ used in the nonlinear terms $\left(u_{r} \frac{\partial u}{\partial x}\right.$ etc.) by using the gradient across the grid box to extrapolate the radar winds out to the vertices. We can then recalculate the momentum balance. Also, as discussed earlier we can use corrected wind values and smoothed satellite fields as further checks. 
3.2.1 Birdlings Flat. The results for the zonal momentum balance above Birdlings Flat using the uncorrected radar zonal wind are displayed in Table 2 . We can see that the Birdlings Flat result for the residual $D$ is -67 $\mathrm{m} \mathrm{s}^{-1} \mathrm{~d}^{-1}$, with the range being -28 to -91 in the vicinity. An examination of the $\Phi_{x}$ terms shows that there is a considerable gradient in this quantity in the vicinity of Birdlings Flat, which means that there ought to be a considerable gradient in $-f v$. In an effort to estimate the effect of that gradient on our results, we repeated the calculation using values of the radar meridional wind $v_{r}$ scaled to each corner of the grid using the gradient across the grid. When we do this, we get $D=-68 \mathrm{~m} \mathrm{~s}^{-1} \mathrm{~d}^{-1}$ with a range of -40 to -87 .

The next test we carried out was the effect of the noise in the geopotential grids. That calculation yielded values of $D=-64 \mathrm{~m} \mathrm{~s}^{-1} \mathrm{~d}^{-1}$ with a range of -29 to -85 . The final test was to use the Birdlings Flat radar wind corrected using the regression formula and the smoothed satellite data. In this case we obtained $D=-73 \mathrm{~m} \mathrm{~s}^{-1} \mathrm{~d}^{-1}$ with a range of -35 to -98 .

The results for the meridional momentum balance using the uncorrected radar zonal wind are displayed in Table 3. We can see that the Birdlings Flat result for the residual $E$ is $204 \mathrm{~m} \mathrm{~s}^{-1} \mathrm{~d}^{-1}$, with the range being 168 to 228 in the vicinity.

It is obvious from this table that the most important contributor to the result is the mismatch between the Coriolis torque on the zonal wind $\left(f u_{r}\right)$ and the meridional gradient of geopotential $\left(\Phi_{y}\right)$. This suggests that the most important contributor to the $E$ term might be inaccuracies (if they exist) in the radar wind.

Accordingly, we present the same table recalculated with the corrected radar zonal wind using the regression line discussed as Table 4. As might be expected, this change drops the residual significantly to $E=56$ $\mathrm{m} \mathrm{s}^{-1} \mathrm{~d}^{-1}$ with a range of 10 to 86 .

As in the case of the zonal momentum balance, we can further examine the sensitivity of this calculation, modifying the use of the winds at the corners and using the smoothed geopotential fields. When we do this, we obtain $E=42 \mathrm{~m} \mathrm{~s}^{-1} \mathrm{~d}^{-1}$ with a range of 11 to 58 .

Although the time tendencies have been neglected, a backward difference calculation using the mean winds at Birdlings Flat yields a monthly mean value of $\langle\partial u / \partial t\rangle=1 \mathrm{~m} \mathrm{~s}^{-1} \mathrm{~d}^{-1}$ and $\langle\partial v / \partial t\rangle=0 \mathrm{~m} \mathrm{~s}^{-1} \mathrm{~d}^{-1}$ with standard deviations of $\sigma_{u_{r}}=14$ for the zonal wind and $\sigma_{v_{r}}=12$ for the meridional. It is more difficult to assess the effect of neglecting the vertical wind terms, however, our assumption that they are negligible is borne out by the results of Marks (1989) whose calculations using a radiative code and climatological data yielded values of $0 \pm 0 \times 10^{-5} \mathrm{~m} \mathrm{~s}^{-1}$ for the Northern Hemisphere winter vertical advection terms in both the zonal and meridional momentum balance.

3.2.2 Adelaide. The same procedures were followed with radar data from Adelaide, along with appropriate ISAMS data. The zonal momentum balance for Adelaide is displayed in Table 5. Here we see a value of $D=29 \mathrm{~m} \mathrm{~s}^{-1} \mathrm{~d}^{-1}$ with a range from -78 to 172 . It is immediately obvious that at this location the balance is predominantly between the curvature of the zonal wind $u_{r} \partial u_{g} / \partial x$ and the geopotential gradient $\Phi_{x}$. It is unfortunate that at this location and for this month these are two large similar-sized numbers, and so we can have little confidence in the value of $D$ obtained. Tests with the smoothed fields give similar results, and
Table 2. Birdlings Flat zonal momentum balance (Eq. 1) in ted radar winds $\left(u_{r}=10\right)$ $\mathrm{m} \mathrm{s}^{-1} \mathrm{~d}^{-1}$ using the uncorrec-
Table 3. Birdlings Flat Meridional momentum balance (Eq. 2) in $\mathrm{m} \mathrm{s}^{-1} \mathrm{~d}^{-1}$ using the uncorrected radar winds $\left(u_{r}=10\right)$

\begin{tabular}{lllllllll}
\hline$\phi$ & $\lambda$ & $\partial u / \partial t$ & $u_{r} \partial u_{g} / \partial x$ & $v_{r} \partial c u_{g} / \partial y$ & $w \partial u / \partial z$ & $\Phi_{x}$ & $-f v_{r}$ & $D$ \\
\hline-42.5 & 170 & 0 & -14 & -1 & 0 & -102 & 25 & -91 \\
-42.5 & 180 & 0 & -5 & -1 & 0 & -58 & 25 & -38 \\
-47.5 & 170 & 0 & -3 & -1 & 0 & -62 & 28 & -36 \\
-47.5 & 180 & 0 & -6 & 0 & 0 & -50 & 28 & -28 \\
-43.8 & 173 & 0 & -10 & -1 & 0 & -82 & 26 & -67 \\
\hline
\end{tabular}

\begin{tabular}{llllllllll}
\hline$\phi$ & $\lambda$ & $\partial v / \partial t$ & $u_{r}^{2} t$ & $u_{r} \partial v_{g} / \partial x$ & $v_{r} \partial v_{g} / \partial y$ & $w \partial v / \partial z$ & $\Phi_{y}$ & $f u_{r}$ & $E$ \\
\hline-42.5 & 170 & 0 & -1 & -5 & -2 & 0 & 321 & -85 & 228 \\
-42.5 & 180 & 0 & -1 & -16 & -5 & 0 & 274 & -85 & 168 \\
-47.5 & 170 & 0 & -1 & -11 & -3 & 0 & 290 & -93 & 182 \\
-47.5 & 180 & 0 & -1 & -5 & 2 & 0 & 268 & -93 & 180 \\
-43.8 & 173 & 0 & -1 & -8 & -2 & 0 & 302 & -86 & 204 \\
\hline
\end{tabular}

Table 4. Birdlings Flat Meridional momentum balance (Eq. 2) in $\mathrm{m} \mathrm{s}^{-1} \mathrm{~d}^{-1}$ using the corrected radar winds $\left(u_{r}=25\right)$

\begin{tabular}{llllllllll}
\hline$\phi$ & $\lambda$ & $\partial v / \partial t$ & $u_{r}^{2} t$ & $u_{r} \partial v_{g} / \partial x$ & $v_{r} \partial v_{g} / \partial y$ & $w \partial v / \partial z$ & $\Phi_{y}$ & $f u_{r}$ & $E$ \\
\hline-42.5 & 170 & 0 & -8 & -13 & -2 & 0 & 321 & -212 & 86 \\
-42.5 & 180 & 0 & -8 & -40 & -5 & 0 & 274 & -212 & 10 \\
-47.5 & 170 & 0 & -9 & -26 & -3 & 0 & 290 & -231 & 19 \\
-47.5 & 180 & 0 & -9 & 13 & 2 & 0 & 268 & -231 & 42 \\
-43.8 & 173 & 0 & -8 & -20 & -2 & 0 & 302 & -216 & 56 \\
\hline
\end{tabular}


Table 5. Adelaide zonal momentum balance (Eq. 1) in $\mathrm{m} \mathrm{s}^{-1} \mathrm{~d}^{-1}$ (here $v_{r}=0.05 \mathrm{~m} \mathrm{~s}^{-1}$ and $u_{r}=29 \mathrm{~m} \mathrm{~s}^{-1}$ )

\begin{tabular}{llllllrlr}
\hline$\phi$ & $\lambda$ & $\partial u / \partial t$ & $u_{r} \partial u_{g} / \partial x$ & $v_{r} \partial c u_{g} / \partial y$ & $w \partial u / \partial z$ & $\Phi_{x}$ & $-f v_{r}$ & $D$ \\
\hline-32.5 & 130 & 0 & -43 & 0 & 0 & -34 & 0 & -78 \\
-32.5 & 140 & 0 & -53 & 0 & 0 & -3 & 0 & -56 \\
-37.5 & 130 & 0 & 29 & 0 & 0 & 143 & 0 & 172 \\
-37.5 & 140 & 0 & 44 & 0 & 0 & 66 & 0 & 110 \\
-35 & 138 & 0 & -5 & 0 & 0 & 34 & 0 & 29 \\
\hline
\end{tabular}

Table 6. Adelaide Meridional momentum balance (Eq. 2) in $\mathrm{m} \mathrm{s}^{-1} \mathrm{~d}^{-1}$

\begin{tabular}{lllllllllr}
\hline$\phi$ & $\lambda$ & $\partial v / \partial t$ & $u_{r}^{2} t$ & $u_{r} \partial v_{g} / \partial x$ & $v_{r} \partial v_{g} / \partial y$ & $w \partial v / \partial z$ & $\Phi_{y}$ & $f u_{r}$ & $E$ \\
\hline-32.5 & 130 & 0 & -7 & -21 & 0 & 0 & 83 & -193 & -138 \\
-32.5 & 140 & 0 & -7 & 65 & 0 & 0 & 18 & -193 & -112 \\
-37.5 & 130 & 0 & -9 & -84 & 0 & 0 & 241 & -219 & -70 \\
-37.5 & 140 & 0 & -9 & 11 & 0 & 0 & 267 & -219 & 51 \\
-35 & 138 & 0 & -8 & 29 & 0 & 0 & 144 & -206 & -41 \\
\hline
\end{tabular}

it is not possible to conclude that $D$ is demonstrably non-zero at Adelaide. However, it is possible to conclude that geostrophy is definitely not a useful concept at $80 \mathrm{~km}$ above Adelaide in May 1992.

The calculation of the meridional momentum balance (Table 6) shows that the balance is primarily between the Coriolis torque on the zonal wind $\left(f u_{r}\right)$ and the geopotential gradient $\left(\Phi_{y}\right)$ although the curvature term $\left(v_{r} \partial v_{g} / \partial y\right)$ can be important too. In this case $E=-41 \mathrm{~m} \mathrm{~s}^{-1} \mathrm{~d}^{-1}$ with a range of 51 to -138 . This value of $E$ is also not demonstrably non-zero, as there is still an extreme gradient of these quantities in the neighbourhood of Adelaide. However, the recalculation with smoothed geopotential gradients and using the modified winds at the corner points gives $E=-54 \mathrm{~m} \mathrm{~s}^{-1} \mathrm{~d}^{-1}$ with a range of 15 to -112 , which implies that as the fields are smoothed, the range of values indicates the probability that $E$ is large and negative.

\section{Discussion}

Before discussing the geophysical significance of the estimates of $D$ and $E$ it is important to consider whether we should have any confidence in these calculations at all. Clearly the calculations are very sensitive to the values of the radar winds and to the gradients in the satellite derived quantities. Regrettably, we must have caveats about both of these data sets, as will be discussed. However, the method we have demonstrated could be used with other data in which one might have more confidence, and with future instruments.

Apart from faults in our assumptions, the two major problems which could impact on our results are whether or not the radar wind is the true wind, and whether or not the gradients we have obtained from satellite are good or not. Regrettably the ISAMS data from May 1992 is rather noisy due to problems with the hardware, and we believe this is the origin of large variations from day-to-day in the curvature terms (and in the standard deviations of $u_{q}$ and $v_{q}$ shown in Table 1). We hope that our averaging has removed most of this ungeophysical noise. Further, even when the data are good, it is possible that the gradient terms are biased due to the way the temperature retrieval works at these altitudes. Thus there is potentially a further unquantifiable error in our calculations.

As far as the radar winds go, at first glance the "corrected" radar winds give the more plausible results at Birdlings Flat because the regression line obtained from comparisons with HRDI leads to larger values of $D$ and smaller values of $E$. However it is not obvious that this is evidence for the HRDI winds being "correct", as the difference between the two sets of winds could be due to local gravity wave driving from the mountains below.

A priori one might imagine that gravity waves propagating in the east-west direction have a better chance of reaching the altitude of $80 \mathrm{~km}$ (as waves propagating in the meridional direction are more likely to encounter critical levels). However, it is important to remember that the critical level is actually encountered when the wave-phase velocity is equal to the projection of the background wind in the direction of the phase velocity (not necessarily when any individual components are equal). Thus it would seem plausible that waves being generated from or near the Southern Alps below (which are orientated from the northeast to the southwest) could reach these altitudes in May (when the winter circulation patterns are established) and lead to large components in either direction. Given that one of the reasons why the HRDI winds and the radar winds differ could be the intense localization of these phenomena (as opposed to errors in either technique), it can only really be stated that the corrected winds may give a better picture of the large-scale forcing terms, while the uncorrected winds may give a better picture of the local forcing. This is tantamount to saying the magnitude of the local forcing due to orography at Birdlings Flat could be as much as $200 \mathrm{~m} \mathrm{~s}^{-1} \mathrm{~d}^{-1}$ in the meridional direction and is only around $60 \mathrm{~m} \mathrm{~s}^{-1} \mathrm{~d}^{-1}$ in the zonal direction.

This argument might also contribute to the inconclusiveness of the Adelaide results. Given that the orography in the neighbourhood of Adelaide is not 
significant (at least in comparison to the Southern Alps), the drag terms there are probably dominated by gravity waves from other sources, and are simply not large enough to be significant in comparison to the curvature terms (at least for that month).

This is not the first study to compare satellite and radar winds. Labitzke et al. (1987) in a comparison of MF radar and winds derived from Stratospheric and Mesospheric Sounder (SAMS) temperatures, found significant ageostrophy in the winter months, in accordance with the results presented here. Manson et al. (1991) used a detailed analysis of radar data from a number of sites to show possible shortcomings in the satellite based CIRA-86 model (CIRA: COSPAR International Reference Atmosphere). They also noted very good balance between the Coriolis torque on the mean flow and the divergence of the vertical flux of horizontal momentum which was measured using radars at a number of sites.

Where our work differs from those previous studies is in the quantitative calculations which are possible with the ISAMS data. In our work, the divergence of the vertical flux of zonal and meridional momentum can be equated to $D$ and $E$ and respectively. With knowledge of the local geopotential gradients, it is clear from either Table 2 or 5 that the balance between the Coriolis torque on the meridional flow and the zonal forcing term is not nearly as good as that reported by other workers, at least at this height and time. (It should be noted however, that in the zonal mean the zonal derivative of the geopotential gradient must disappear, and so the Coriolis torque on the zonal mean meridional wind must balance the zonal mean gravity wave term $D$ and or the curvature term.)

The importance of the curvature and residual terms in momentum balance is tantamount to saying that geostrophic balance is not a useful concept at these locations, altitudes and time. This is most obvious above Adelaide where it is apparent that the curvature terms are an important contributor to the balance with the geopotential gradients, particularly in the zonal momentum equation where the Coriolis torque is clearly not significant (because of the weak meridional flow). It is important to note that this result is independent of whether or not the radar measures accurate winds and arises simply because the curvature terms are very large.

The actual value of $D$ obtained at Birdlings Flat where we have some confidence in the result is around $60 \mathrm{~m} \mathrm{~s}^{-1} \mathrm{~d}^{-1}$, which is consistent with those found using; radars (Manson et al., 1991; Fritts and Vincent, 1987), CIRA data Marks (1989); Medvedev and Fomichev (1994), satellites (e.g. Fetzger and Gille, 1996), and theory (e.g. Holton, 1983).

As far as we are aware this technique provides the first estimates of the magnitude of the meridional forcing term which are not based directly on radar measurements of the divergence of the vertical flux of meridional momentum. Examination of Tables 3 and 4 shows that $E$ is mainly dependent on the zonal wind via the Coriolis torque term and also the zonal advection of meridional momentum. It is thus possible that the resultant values of $E$ are strongly biased by systematic errors (if they exist) in the zonal wind.

Despite the difference between the results using the actual radar wind (Table 3) and the "corrected" radar wind (Table 4), it is clear that at least at Birdlings Flat there is significant local forcing of the flow, which means that the assumption that $E$ is a small part of the momentum balance is incorrect.

Acknowledgements. The authors are grateful to Wayne Hocking for a careful and constructive reading of this work. DJF acknowledges funding from the New Zealand Public Good Science Fund which allowed him to visit Oxford to begin this work.

Topical Editor F. Vial thanks A.K. Smith and another referee for their help in evaluating the paper.

\section{References}

Burrage, M. D., W. R. Skinner, D. A. Gell, P. B. Hays, A. R. Marshall, D. A. Ortland, A. H. Manson, S. J. Franke, D. C. Fritts, P. Hoffman, C. McLandress, R. Niciejewski, F. J. Schmidlin, G. G. Shepherd, W. Singer, T. Tsuda, and R. A. Vincent, Validation of mesosphere and lower thermosphere winds from the high resolution Doppler imager on UARS, J. Geophys. Res., 101, 10 365-10 392, 1996.

Dudhia, A., and N. Livesey, Validation of temperature measurements from the improved stratosphere and mesosphere sounder, J. Geophys. Res., 101, 9795-9809, 1996.

Fetzger, E. J., and J. C. Gille, Gravity wave variance in LIMS temperatures. Part II: comparison with zonal-mean momentum balance, J. Atmos. Sci., 53, 398-410, 1996.

Frame, D. J., B. N. Lawrence, G. J. Fraser, and M. D. Burrage, A comparison between mesospheric wind measurements made near Christchurch (44S, 173E) using the High Resolution Doppler Imager (HRDI) and a medium frequency (MF) radar, Submitted to Ann. Geophys., 1999.

Fritts, D. C., and R. A. Vincent, Mesospheric momentum flux studies at Adelaide, Australia: observations and a gravity wavetidal interaction model, J. Atmos. Sci., 44, 605-619, 1987.

Hamilton, K., Diagnostic study of the momentum balance in the northern hemisphere winter stratosphere, Mon. Weather Rev., 111, 1434-1441, 1983.

Holton, J. R., The influence of gravity wave breaking on the general circulation of the middle atmosphere, J. Atmos. Sci., 40, 2497-2507, 1983.

Khattatov, B. V., M. A. Geller, V. A. Yudin, P. B. Hays, W. R. Skinner, M. D. Burrage, S. J. Franke, D. C. Fritts, J. R. Isler, A. H. Manson, C. E. Meek, R. McMurray, W. Singer, P. Hoffman, and R. A. Vincent, Dynamics of the mesosphere and lower thermosphere as seen by MF radars ad by the HighResolution Doppler Imager/UARS, J. Geophys. Res., 101(D6), 10 393-10404, 1996.

Labitzke, K., A. Manson, J. Barnett, and M. Corney, Comparison of geostrophic and observed winds in the upper mesosphere over Saskatoon, Canada, J. Atmos. Terr. Phys., 49, 987-997, 1987.

Lindzen, R. S., Turbulence and stress owing to gravity wave and tidal breakdown, J. Geophys. Res., 86, 9707-9714, 1981.

Manson, A., C. Meek, E. Fleming, S. Chandra, R. Vincent, A. Phillips, S. Avery, G. Fraser, M. Smith, J. Fellous, and M. Massebeuf, Comparisons between satellite-derived gradient winds and radar-derived winds from CIRA-86, J. Atmos. Sci., 48, 411-428, 1991.

Marks, C. J., Some features of the climatology of the middle atmosphere revealed by Nimbus 5 and 6, J. Atmos. Sci., 46, 2485-2508, 1989.

Medvedev, A., and V. Fomichev, Net radiative heating and diagnostics of the diabatic circulation in the $15-110 \mathrm{~km}$ height layer, J. Atmos. Terr. Phys., 56, 1571-1584, 1994. 
Randel, W. J., The evaluation of winds from geopotential height data in the stratosphere, J. Atmos. Sci., 44, 3097-3120, 1987.

Rosier, S., B. Lawrence, D. Andrews, and F. Taylor, Dynamical evolution of the northern stratosphere in early winter 1991/92, as observed by the improved stratospheric and mesospheric sounder, J. Atmos. Sci., 51, 2783-2799, 1994.
Smith, A. K., and L. V. Lyjak, An observational estimate of gravity wave drag from the momentum balance in the middle atmosphere, J. Geophys. Res., 90, 2233-2241, 1985.

Swinbank, R., and A. O'Neill, A stratosphere-troposphere data assimilation system, Mon. Weather Rev., 122, 686-702, 1994. 